\title{
Analysing epilepsy monitoring in Al Ain, UAE
}

\begin{abstract}
Background: Single-bedded inpatient Epilepsy Monitoring Unit (EMU) was started in Al Ain Hospital in July 2016. Epilepsy monitoring is useful in intractable epilepsy for studying electrical origin of seizures, monitoring the number of Interictal Epileptiform Discharges (IEDs) in response to medications and differentiating true epileptic events from psychogenic/physiologic non-epileptic events.
\end{abstract}

Aims: To highlight our experience at Al Ain Hospital- EMU and to increase the awareness about the usefulness of Epilepsy monitoring units in the diagnosis and management of epilepsy and non-epileptic events.

Methods: Retrospective data was analysed from the EMU registry from July 2016 to July 2017. Demographics, duration of monitoring, number of recordings with abnormal IEDs, number of patients with captured events and the type of events were analyzed. We also looked at number of patients with major change in their antiepileptic drug therapy as well as those referred to epilepsy surgery centers after the inpatient video -EEG monitoring.

Results: Sixty patients were admitted during the study period. Thirty one $(n=31)$ were male $\&$ thirty two $(n=32)$ were female. Age range varied from two to sixty seven. Majority of the patients; Forty seven patients were monitored for 3 days. Sixty percent of patients had IEDs in the recording. $38 \%$ patients had epileptic seizures, while $8 \%$ patients had psychogenic non-epileptic events. Out of twenty patients with evaluation for seizures, five patients were referred for epilepsy surgery with focal and intractable seizures.

Most of the patients were admitted for monitoring for a total of 2.5 days ( $n=54$ hours) to 2.7 days ( $\mathrm{n}=57$ hours). EMU was evaluated by dedicated technologists and nurses. Patient safety measures and clear protocols are in place. Our results show that we were able to localize the origin of seizures, aid in adjusting medications and identify the patients for referral to epilepsy surgery in addition to diagnosing non-epileptic events,

Discussion and Conclusion: Our data show EMU monitoring is an extremely useful investigation in management of epilepsy and in diagnosis of non-epileptic events. We have the potential to expand into a 3-bedded unit with adequate availability of resources. We also report that three day monitoring may be sufficient in most cases.

Keywords: epilepsy monitoring unit, epilepsy surgery and,psychogenic non epileptiform events
Volume 8 Issue 4 - 2018

\author{
Syed MF Zabiullah,' Renganathan \\ Radhakrishnan, ${ }^{2}$ Niles h P Baksh, ${ }^{3}$ Vishnu \\ Sudeesh, ${ }^{3}$ Zubeda Mohiuddin,' Khurram A \\ Siddiqui ${ }^{4}$ \\ IInternal Medicine Resident, Department of Academic Affairs, \\ UAE \\ ${ }^{2}$ Consultant Neurologist \& Neurophysiologist, UAE \\ ${ }^{3}$ Division of Neurophysiology, Department of Neurology, UAE \\ ${ }^{4}$ Consultant Neurologist \& Epileptologist, UAE
}

Correspondence: Syed Mohammed Faizaan Zabiullah,

Department of Academic Affairs, Internal Medicine Resident, Al

Ain Hospital, Al Ain, UAE, Tel +97|37022000,

Emailszabiullah@seha.ae

Received: April 19,2018| Published: July 03, 2018
Abbreviations: EMU, epilepsy monitoring unit; PNEA, psychogenic non epileptiform attacks; AED, anti-epileptic drug; EEG, electroencephalogram; PVEEG, prolonged video electroencephalogram; UAE, united arab emirates; GCC, gulf cooperation council; IED, inter-ictal epileptiform discharges

\section{Introduction}

Epilepsy is a widespread, morbid and a very expensive condition According to WHO, It is estimated that worldwide about 50 million people suffer from epilepsy. ${ }^{1}$ There are no prevalence studies conducted in the UAE to estimate population with epilepsy, but estimates in 2014 were around $3 \%$ of general population i.e. 120, 000 people. $^{2}$ Epilepsy/ Video-monitoring in the EMU can help us in identifying seizures from seizure mimics. ${ }^{3}$ Studying the seizure semiology can establish the type of seizure; focal, generalized or focal seizure with secondary generalization. It also helps in diagnosing 'difficult to identify' seizures such as absence seizures and complex partial seizures. ${ }^{4}$ It is also useful in localization of any epileptogenic foci, which may help us in identifying patients for epilepsy surgery. Imaging can also supplement the localization. As such, it is a part of Pre-surgical workup for patients who are candidates for epilepsy surgery. ${ }^{5}$ Epilepsy is also a highly misdiagnosed condition, ${ }^{3}$ as it requires reliable history from the patients and their family members. Many patients with Psychogenic non -epileptic attacks (PNEA) are misdiagnosed as having seizures and a few patients with seizures are misdiagnosed as having PNEA. ${ }^{6}$ The latter is especially true with difficult to diagnose seizures such as temporal lobe epilepsy, frontal lobe seizures or absence seizures. ${ }^{6}$

Routine outpatient EEG is used to identify the type of epilepsy, but it has low sensitivity. ${ }^{7}$ As a result, Epilepsy monitoring units are established to witness a seizure first hand by health professionals and establish the diagnosis. Also, an additional advantage with VideoEEG monitoring is that we can induce seizure under supervision of medical professionals. ${ }^{8,9}$ The value of this is that we can prevent and respond to any adverse events. ${ }^{9}$ Video - EEG in an inpatient EMU can also enable us to use more electrodes for inferior temporal electrodes which can access hard to reach areas not possible on a routine outpatient EEG. ${ }^{10}$ Non seizure artefacts which may appear as abnormal epileptiform readings on EEG tracing can be identified precisely with help of Video EEG. ${ }^{4}$

Epilepsy surgery is being increasingly used for patients having intractable epilepsy and patients having recurrent focal seizures. It is estimated that out of 3 million adult patients suffering from epilepsy, $30 \%$ patients with focal seizures are unresponsive to 
Anti-Epileptic Drugs (AEDs). Half of these patients are candidates for epilepsy surgery. $15 \%$ of patients with generalized epilepsy are pharmacoresistant and are candidates for alternative treatments such as Vagal Nerve Stimulation and palliative epilepsy surgery. ${ }^{11}$ Majority of patients who are candidates for epilepsy surgery are referred outside the UAE for treatment. ${ }^{12}$

Our EMU is a single patient bed facility. Patients are given information leaflets about the EMU, seizure provocation, possible complications, safety and emergency measures in place. An informed consent is obtained for every patient who is admitted. Patients are attached to EEG electrodes and are monitored by continuous video monitoring by a technician and nurse at the monitoring station which is close to the patient's room. Patient and his attendant are educated about the 'nurse call' button in case patient has an aura or a seizure. Each patient has an IV access on admission and facility for suction and oxygen supplementation is present at the head of the bed, to give medical care when patient is having a seizure.

\section{Materials and methods}

A retrospective analysis was conducted on all patients who were admitted to EMU between July 2016 and July 2017. Data was collected from electronic files of the patients from our hospital information system (Cerner). We collected details of patient demographics which included age, nationality, and gender. We also recorded duration of monitoring (in days) and the type of seizure provocation methods employed. We analysed the number of recordings with abnormal IEDs, number of patients with captured events and the type of events.

Our data collection tool collected the following parameters:

i. Number of patients with major change in their antiepileptic drug therapy

ii. Number of patients referred to epilepsy surgery.

iii.Type of first time AED therapy after evaluation in the EMU.

iv. Complete discontinuation of previous AED therapy (eg. in cases of PNEA)

v. AED withdrawal

vi.Photic stimulation

vii. Sleep deprivation

viii. Hyperventilation

We analysed the seizures which were reproduced, the type of the seizures and the localization of the seizure wherever identified. We looked at the number of patients who were identified as true seizures and those with seizure mimics. We also counted the number of patients with seizure mimics in whom AED therapy was stopped. Of the patients in whom seizures were captured, we identified the patients who were candidates for epilepsy surgery. Of these patients who were referred for surgery, we analysed the type of seizure they had.

\section{Results}

A total of 63 patients were admitted to the EMU during our study period. 32 were male and 31 were female.

Age range varied between 2 to 67 years of age. There were $49 \%$ female $(n=31)$ and $51 \%$ male $(n=32)$ with a mean age of 10.5 and standard deviation of \pm 5.68 with a range of 2 to 67 year old $(n=63)$, (Table 1A-1C) (Table 2). Majority of the patients i.e.; $90 \%$ were UAE Nationals $(n=56)$ while six patients were from other GCC countries and other nationalities. Most of the patients were in age group 20 to 30 $(n=20)$. Seven patients were in the age group 0 to 10 , fourteen patients were in the age group 10 to 20 , Nine Patients were in the age groups 30 to 40 , and nine patients were in age group 40 to 50 . Four patients were above the age of $50.90 \%$ patients (56 out of 63 ) were already on AEDs prior to the EMU admission. Only seven did not have any treatment with AED before. Majority of the patients (47 out of 63) spent up to 3 days (54 to 57 hours) in the EMU. Ten Patients spent 2 days, four patients spent 4 days and 2 patients spent 5 days in the EMU, (Figure 1) (Figure 2). One patient spent a few hours as he had a seizure episode within few hours of admission.

Table IA Age group of patients

\begin{tabular}{ll}
\hline Age group & No. of patients \\
\hline 0 to 10 & 7 \\
10 to 20 & 14 \\
20 to 30 & 20 \\
30 to 40 & 9 \\
40 to 50 & 9 \\
$>50$ & 4 \\
total & 63 \\
\hline
\end{tabular}

Table IB Patients Female and Male

\begin{tabular}{lll}
\hline Female & Male & Total \\
\hline 31 & 32 & 63 \\
$49.21 \%$ & $50.79 \%$ & $\mathrm{I}$
\end{tabular}

Table IC Different Nationals

\begin{tabular}{lll}
\hline Nationality & Number of pts. & Percentage \\
\hline UAE & 57 & $90.48 \%$ \\
GCC & 3 & $4.76 \%$ \\
non GCC & 3 & $4.76 \%$ \\
total & 63 & $100.00 \%$ \\
\hline
\end{tabular}

Table 2 Provocation of Seizures

\begin{tabular}{ll}
\hline Seizure inducing methods & no. of patients \\
\hline Withholding AED & 49 \\
Sleep Deprivation & 57 \\
hyperventilation & 58 \\
photic stimulation & 59
\end{tabular}

Most patients had previous workup for epilepsy which included routine EEG, MRI and even PV-EEG. Sixty one patients had a previous routine EEG done. Only 2 patients did not have any previous EEG. Six patients had previous video- EEG monitoring as well. Barring 1, all the patients $(\mathrm{n}=62)$ had a brain MRI with and without contrast. Seizure provocation methods while in EMU included reducing and holding AEDs within 24 hours, photic stimulation, hyperventilation and sleep deprivation. Most of the patients were subjected to all these 
measures i.e - AEDs were withheld in 49 patients, 57 patients were subjected to sleep deprivation, 59 patients were subjected to photic stimulation, and 58 patients underwent hyperventilation. As we can see, most patients $(n=49)$ had a combination of all the four methods of seizure provocation. Out of the total of 63 patients, IEDs were detected in 41 patients which are $65 \%$ of the total patients. True seizures were detected in 20 patients: $11(17 \%)$ had focal seizures and $9(14 \%)$ had generalized seizures. 5 patients $(8 \%)$ had PNEA, 1 patient had hypersomnia and 37 patients (59\%) did not have any seizure activity.

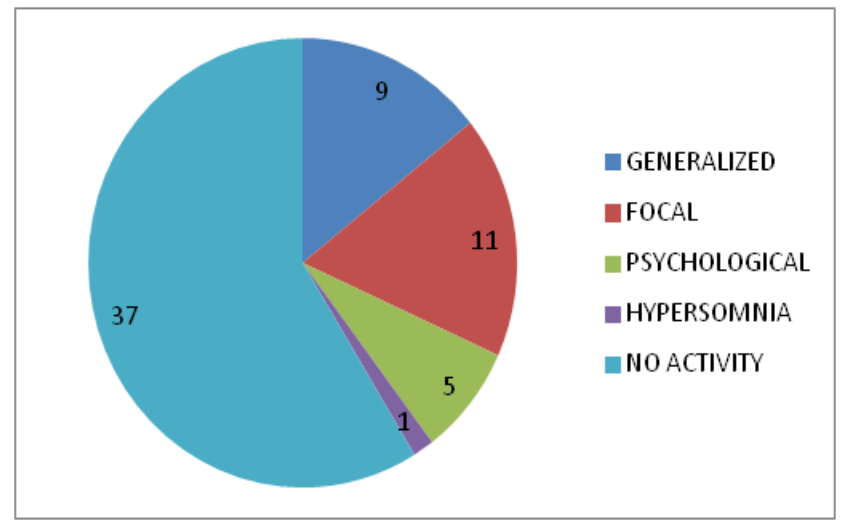

Figure I Types of Seizure.

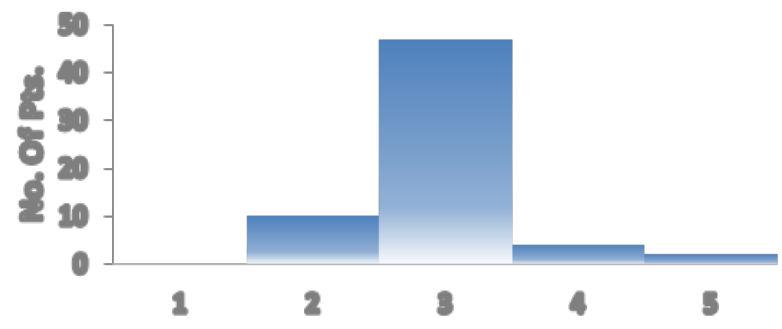

Figure 2 Days in EMU.

\section{Discussion and analysis}

The EMU is part of a comprehensive epilepsy program, in which patients are evaluated with a number of tests. One of the main goals is identification of epileptic paroxysmal electrographic abnormalities. These include seizures (overt, clinical and subclinical) and interictal epileptiform discharges. The other important goal is identification of behavioural abnormalities which are wrongly diagnosed as seizures. Based on the results of our study, an EMU allows us to determine 2 important categorical aspects of Epilepsy among others:

1. Diagnosis of paroxysmal non-epileptic events.

\section{Proper diagnosis of the epilepsy syndrome.}

After evaluation in an EMU, we can place patients in one of the above categories which can assist us to determine and optimize management for patients with Epilepsy i.e. whether or not to start/ continue AEDs, refer for epilepsy surgery, lifestyle changes, diet therapy or use of neurostimulation devices. ${ }^{10-12}$
This is supported by our results as 56 patients ( $89 \%)$ of our patients were prescribed AEDs on discharge, and 6 patients had a major change in their therapy; 3 already on AEDs were asked to stop their AEDs as they were diagnosed with PNEAs while 3 patients were started on AEDs for the first time - all as a result of Epilepsy monitoring. Out of the 20 patients with identified seizures, five patients were identified as potential candidates for epilepsy surgery. Of these five, four had focal seizures and one had intractable primary generalized seizures. All the patients were referred to epilepsy surgery centres outside of the UAE for further management.

During classification; patients with a diagnosis of epilepsy but poor classification of the clinical seizure types, or patients with a diagnosis of epilepsy for further classification of epilepsy syndrome have been evaluated. These patients are evaluated by an Epileptologist either before or during admission to the EMU. The evaluation includes aspects such as drug tapering, deciding on the types of seizures to be recorded, and the optimal number of seizures to be recorded. Other important technical details such as Initiation of recordings, the number of EMG channels, EOG, need for extra electrodes (discussed with the EEG technologist), duration of recordings are also decided by the epileptologist. The epileptologist then reviews the recordings, communicates the results with patients and the family (pediatrics), prepares the video-EEG reports and discharge summaries, in addition to caring for medical issues that may arise during admission. Many of the results are also discussed in seizure conferences to decide the treatment (medical or surgical) and/or referral to a major epilepsy centre. During application of electrodes on the patient (while the patient is seated or lying on bed), standard precautions to prevent accidental falls and injuries are taken. The patient's eyes are covered while applying collodion via air pump to prevent accidental spraying. Electrodes are applied in a systematic fashion to prevent entanglement utilizing cable management techniques like cable ties, wraps and cable tube covers to prevent accidental strangulation during sleep while in the EMU in accordance to hospital's entanglement policies. The electrode amplifier is properly secured and wrapped in a heatproof pouch to prevent accidental burn to the patient or accidental disconnection of electrodes during monitoring. The EMU bed is equipped with proper safety bumper pads to protect the patient during seizures. ${ }^{13-15}$ Mittens are sometimes used with difficult patients to prevent accidental and/or intentional disconnection of electrodes. Use of a head wrap, burn net or conforming bandage to secure electrodes is also common with difficult patients to prevent accidental and/or intentional disconnection of electrodes. If required, daily scalp checks are required to assess for development of pressure sores. Patients on whom medication is being tapered during EMU stay have an IV in place along with an EKG and $\mathrm{O} 2$ saturation monitor. Age appropriate restraints (if needed) are used as required as per hospital's restraint policies. The room is equipped with a 'Nurse Call' button at the bedside, separate from seizure pushbutton. ${ }^{13-15}$

Based on our experience, evidence based data, results of our study and discussion, we have implemented an algorithm on how an EMU should be properly run:

Initiate EMU equipment for continuous video EEG monitoring: Day 1: Following electrode application, a 10-minute baseline EEG should be recorded to test the integrity of the electrode application, during this recording, $\mathrm{HV}$ and photic stimulation may be performed as part of the baseline set-up. Once the patient is settled in the EMU, 
the seizure safety guidelines should be reviewed with the patient and/ or attendant. Patient and/or attendant should be instructed on the importance of completing the seizure log sheet for documentation. The push-button alarm must be tested by the patient and/or caregiver. Both the video and infrared camera (including audio) is tested prior to the start of the EMU recording. The EMU Tech then initiates the EMU equipment for day 1 continuous video EEG monitoring. ${ }^{13-15}$

Review and mark seizures, push-button events and automatic seizure and spike detections for emu epileptologist: Day 2: The EMU Tech checks EMU patients in the morning and rechecks at the end of their shift, reviews patient seizure and push button log, re-gels and/or reapplies any electrodes that have become loose detached or are otherwise not functioning properly. This sequence is carried out every day that the patient is in the EMU, until he or she is discharged. $13-15$

Data management: archival and pruning EEG and video segments for storage: All EEG and video data is reviewed, marked and annotated by the EMU Tech for electroencephalographer or clinical Neurophysiologist to review. Following data interpretation, relevant EEG or entire EEG file and video segments should be archived to centralized server as per naming conventions at the hospital. Data management on recording stations need to be managed as needed (e.g. local copies on acquisition station hard drives may need to be deleted based-on available space) to continue uninterrupted recording and prevent lack of storage space locally. Each EMU acquisition unit must have enough storage space to store data on the local hard drives for a minimum of 5 days. Provide both technical and computer backup for problems that may arise during the monitoring session. EMU Technologists should be assigned to take care of the EMU patients during their stay. It is recommended that an EMU Tech be on-call to provide both technical and computer support to resolve issues during the monitoring session. This support is necessary to prevent data loss and decreased hardware and software downtime during an EMU session when several patients are monitored simultaneously.

Clean and discharge patient: The EMU session is stopped following directive from the clinical team managing the patient (e.g., once the desired number of seizures is captured and the clinical question for the EMU admission has been answered). Using appropriate PPE (preventive protective equipment), electrodes are removed using appropriate techniques (e.g. collodion remover is recommended as a safe acetone-free solution to remove electrodes). ${ }^{13-15}$ This data gives us loads of information which can help us understanding the type of epilepsy and evaluations.

\section{Conclusion}

Our EMU has been evaluated and is planned to expand in future. We had an encouraging year experience at the EMU in $\mathrm{Al}$ Ain region. Since epilepsy is a disease with lifelong implications, we have to be careful in labelling patients as epileptic especially in cases of doubtful history and presentations. In such patients, in addition to establishing the diagnoses, video-EEG monitoring can aid in identifying a seizure and save the patients from complications of long term AED therapy. As is evident from our data, we have managed to clarify the diagnoses, optimize therapy and identify patients for epilepsy surgery referrals in many patients. EMU plays an important role in diagnosing and optimizing management in patients with epilepsy. It is also important in identifying patients for epilepsy surgery. Since the start of the EMU in our hospital, we have managed to clarify the diagnosis of epilepsy in our patients. We have changed the epilepsy management in 11 patients (17.43\% of our patients)- 3 patients newly commenced on AEDs, 3 patients who were wrongly on AEDs were asked to discontinue the AEDs and 5 patients were referred for epilepsy surgery. These are life altering changes as we know that diagnosis of epilepsy and its treatment is a lifelong process. It has major implications on the cost of treatment and quality of life of these patients. This also sheds light on the important aspect of the condition and any misdiagnoses. After this study, we can safely assume that like in the rest of the world, even in UAE, a substantial number of patients are wrongly labelled as having epilepsy. And a detailed evaluation in EMU can reduce this number. Since epilepsy is a huge problem even in UAE, we feel that having more EMUs can effectively improve epilepsy care by reducing misdiagnoses, clarifying the subtype of seizures, localization of the seizures and identifying patients for possible epilepsy surgery. We would like to continue this study by including data from 2017 onwards to date and in future to build up on our statistics. Given proper resources, we can expand our EMU to a higher number of beds from 3 to 5 . This will enable us to increase the number of patients to two and then 4 enabling us to improve the management of epilepsy in Al Ain region, which is part of Abu Dhabi emirate, UAE. We can also enhance the epilepsy management by collaborating with radiologists for functional scans and may be in future lead to establishment of adequate epilepsy surgery services. Most of the patients are sent abroad for epilepsy surgery and improving epilepsy care can substantially reduce the cost of treatment as it can be done locally with better EMU services in the region.

\section{Acknowledgements}

None.

\section{Conflict of interest}

The author declares no conflict of interest.

\section{References}

1. WHO. Epilepsy. WHO. World Health Organization; 2018

2. Emirati league against epilepsy, press release; 2014.

3. Worrell GA, Lagerlund TD, Buchhalter JR, et al. Role and Limitations of routine and ambulatory scalp electroencephalography in diagnosing and managing seizures. Mayo Clin Proc. 2002;77(9):991.

4. Atkinson M, Hari K, Schaefer K, et al. Improving safety outcomes in the epilepsy monitoring unit. Seizure. 2012;21(2):124-127.

5. Kanner AM. Common errors made in diagnosis and treatment of epilepsy. Semin Neurol. 2008;28(3):364-378.

6. Moien-Afshari F, Griebel R, Sadanand V, et al. Safety and yield of early cessation of AEDs in video-EEG telemetry and outcomes. Can J Neurol Sci. 2009;36(5):587.

7. Noe KH, Drazkowski JF. Safety of long term videoelectroencephalographic monitoring for evaluation of epilepsy. Mayo Clin Proc. 2009;84(6):495.

8. Moeller, Jeremy M, Haider HAM, et al. Electroencephalography (EEG) in the diagnosis of seizures and epilepsy; 2018

9. Hirsch, Lawrence JM, Haider HAM, et al. Video and ambulatory EEG monitoring in the diagnosis of seizures and epilepsy; 2017

10. Passaro, Erasmo AM, Benbadis, Selim RM. Identification of potential epilepsy surgery candidates: Overview, medical intractability, consequences of uncontrolled epilepsy. 2014. 
11. Brenner RP, Drislane FW, Ebersole JS, et al. Guideline twelve: guidelines for long-term monitoring for epilepsy. J Clin Neurophysiol. 2008;25(3):170-180.

12. Carlson C. First do no harm: safety in the epilepsy monitoring unit Epilepsy Currents. 2009;9(6):162-163.

13. Chatrian GE. Ten percent electrode system for topographic studies of spontaneous and evoked EEG activity. Am J Electroencephalogr Technol. 1985;25(2):83-92.
14. Dobesberger J, Walser G, Unterberger I, et al. Video-EEG monitoring: safety and adverse events in 507 consecutive patients. Epilepsia. 2011;52(3):443-452.

15. Shafer PO, Buelow J, Ficker DM, et al. Risk of adverse events on epilepsy monitoring units: a survey of epilepsy professionals. Epilepsy Behav. 2011;20(3):502-505. 